\title{
Optical CDMA Networks with Bipolar Codes
}

\author{
A. Gameiro \\ Univ. Aveiro / Instituto Telec., Campus Universitário, 3810-193 Aveiro, Portugal
}

Key words: Optical CDMA, Networks, Spread-Spectrum

\begin{abstract}
In this communication we consider a dual wavelength scheme that allows the use of bipolar codes in optical code division multiple access networks. The performance of the system is derived for asynchronous and asynchronous users, and the results show that significant throughput improvements are achievable relatively to the conventional schemes relying on unipolar codes.
\end{abstract}

\section{INTRODUCTION}

Optical fiber networking will be undoubtedly one of the key components to meet the growing demands of the information society. At the core of the development is the huge bandwidth of a single mode fiber that can support up to several Tbit/s transmission capacity. Due to the speed limitation of signal processing in the electrical domain, optical multiplexing techniques have to be employed to exploit the full transmission capacity.

Presently there is some consensus on the use of wavelength division multiplexing [WDM] for more efficient utilization of the bandwidth of existing optical fiber links, upgrading their capacity in a cost effective manner [1]. The implementation of dense WDM requires however high precision wavelength stabilization techniques as well as the optical frequency standard. An alternative to WDM is code division multiple access (CDMA), or code division multiplexing (CDM), where the signals from different terminals coexist either in time or frequency, and are discriminated through an unique code or signature attributed to each user. CDMA relies on spread spectrum transmission techniques, and has gained a wide acceptance 
in radio communications: it is used in several commercial systems, and has already been specified as the technology for third generation cellular systems. Code division multiplexing offers some advantages over the more traditional techniques based on time or frequency discrimination, namely:

- It allows uncoordinated access to the common transmission medium;

- It presents a soft performance degradation as a function of the number of users, which may be exploited when considering applications requiring different quality of services;

- It presents some inherent security.

These advantages result in a more flexible network requiring a less sophisticated management when compared to FDM or TDM. Furthermore in optical fiber networking CDM can be elegantly overlaid on the existing WDM networks providing an enhanced versatility and helping to solve the future wavelength resource problems [2]. It is therefore expectable that similarly to what happened in radio communications, the CDM techniques will be a key component of optical multiplexing in the medium term, and in fact considerable research on this topic has been carried out in the last years. The work on optical CDMA (OCDMA) has been focused towards two different categories: 1) coherent systems using phase-shifted code sequences [3-7] and 2) non-coherent systems adopting intensity modulated sequences $[2,8-10]$. Non-coherent systems have significant advantages in terms of the complexity required to build the transmitters and receivers, but because they are based on intensity modulation, the signature codes are restricted to unipolar $(0,1)$ sequences. This non-negative nature of the signals, prevents the use of the well studied bipolar codes commonly used in radio communications and provided the impetus for the development of unipolar pseudo-orthogonal codes [3, 5,11-12]. The number of available codes is however rather low and they are clearly inferior in terms of auto-correlation or cross-correlation properties to the well designed bipolar codes such as Gold or Kasami sequences, thus putting limits on the maximum number of simultaneous users. To allow the use of bipolar codes and thus take advantage of the extensive material available on the design of bipolar sequences with good properties, one solution is to go to optical coherent communications. The option for coherent communications represent however a significant increase in the complexity of transceivers: the coders / decoders must employ phase shifters to reverse polarity, and receivers require phase feedback control loops which are difficult to engineer. Motivated by these facts we consider in this communication a solution of intermediate complexity: the coders / decoders are based on phase-shifted optical sequences enabling the use of bipolar codes, but information corresponding to logical ones and logical zeros is sent in different 
wavelengths which are detected non-coherently. This constitutes basically a non-coherent FSK system. The communication is outlined as follows. In section 2 we present the architecture of the system we will be dealing with and describe the transceivers for the non-coherent system suitable to operate with bipolar codes. In sections 3 we derive the performance of the proposal for both cases of synchronous and asynchronous networks, and based on these results present in section 4 numerical examples and comparisons against the corresponding systems based on unipolar codes. Finally in section 5 we outline the main conclusions of this work.

\section{SYSTEM MODEL}

The general architecture of the network we are dealing with is shown in Fig. 1. There are $\mathrm{N}$ transmitter / receiver pairs (users), and each user is assigned a unique signature or code sequence to assess the common medium. The block diagram of the transmitter used at each station is shown in Fig. 2. Two pulsed laser diodes (LD), one operating at wavelength $\lambda_{0}$ and the other at wavelength $\lambda_{1}$ are used. Each LD outputs a train of pulses with duration $\tau$, and period $T$, where $1 / T$ is the data bit rate. The data sequence modulates the intensity of the first LD, and the complementary data the intensity of the second. With a logical 1 in the incoming data we get a pulse of duration $\tau$ at the output of the upper modulator and no signal at the output of the lower,

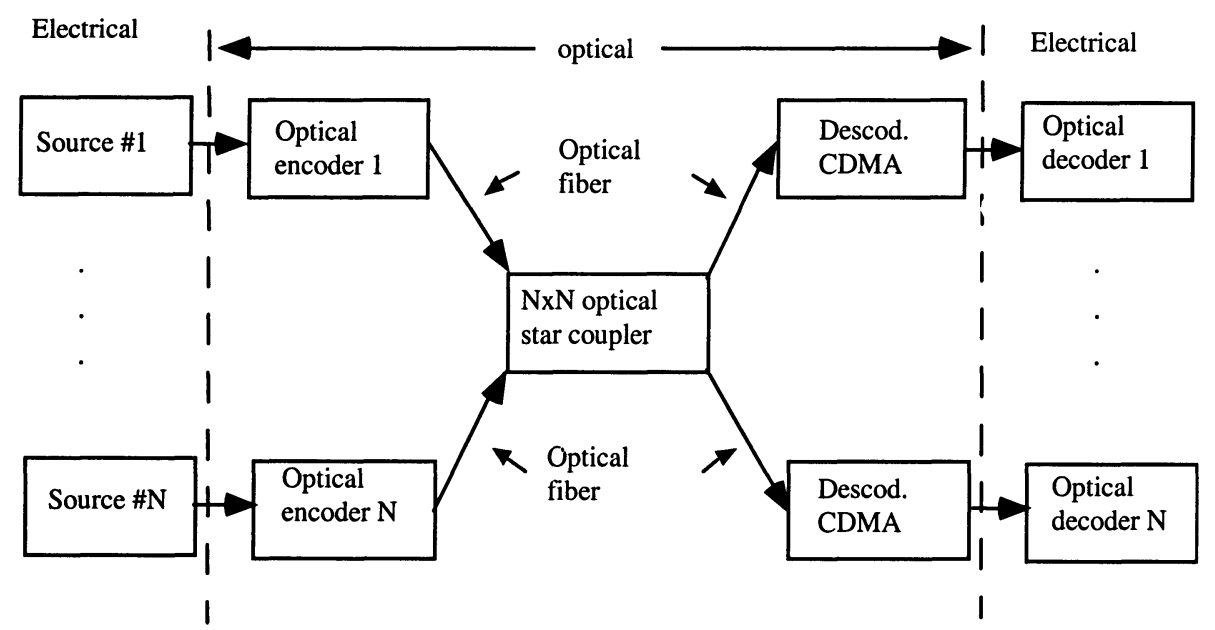

Figure 1. Optical CDMA system in a star configuration. 
while for a logical 0 the situation is reversed. The transmitter encoder may be implemented using the fiber optic transversal filters of Fig. 3, which have been experimentally demonstrated in [2] and [9], although other ladder network structures are also feasible [13]. The phase shifts of the various taps are tuned to be 0 or $\pi$, which allows one to generate with $N$ taps any PSK code of length $N$.

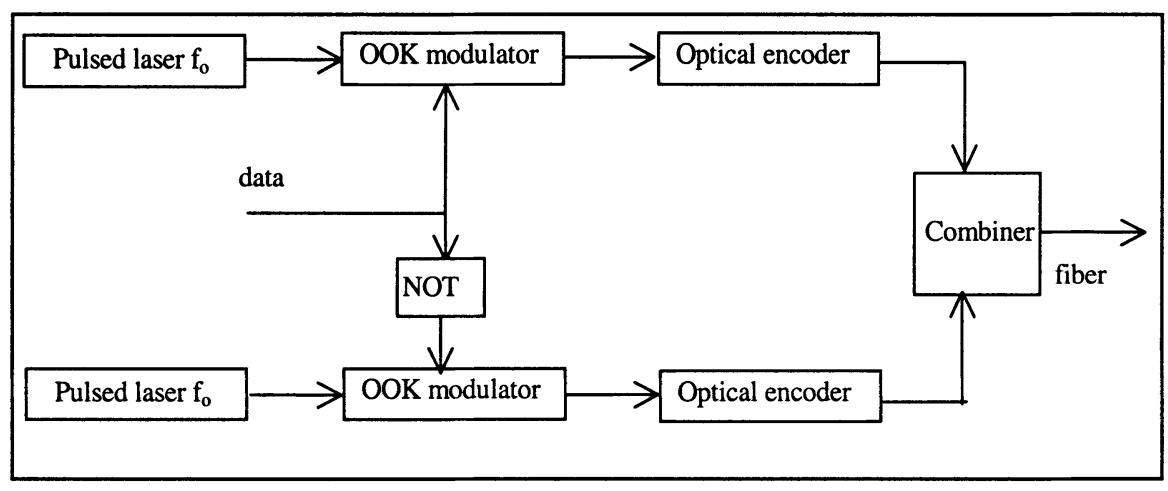

Figure 2. Block diagram of transmitter

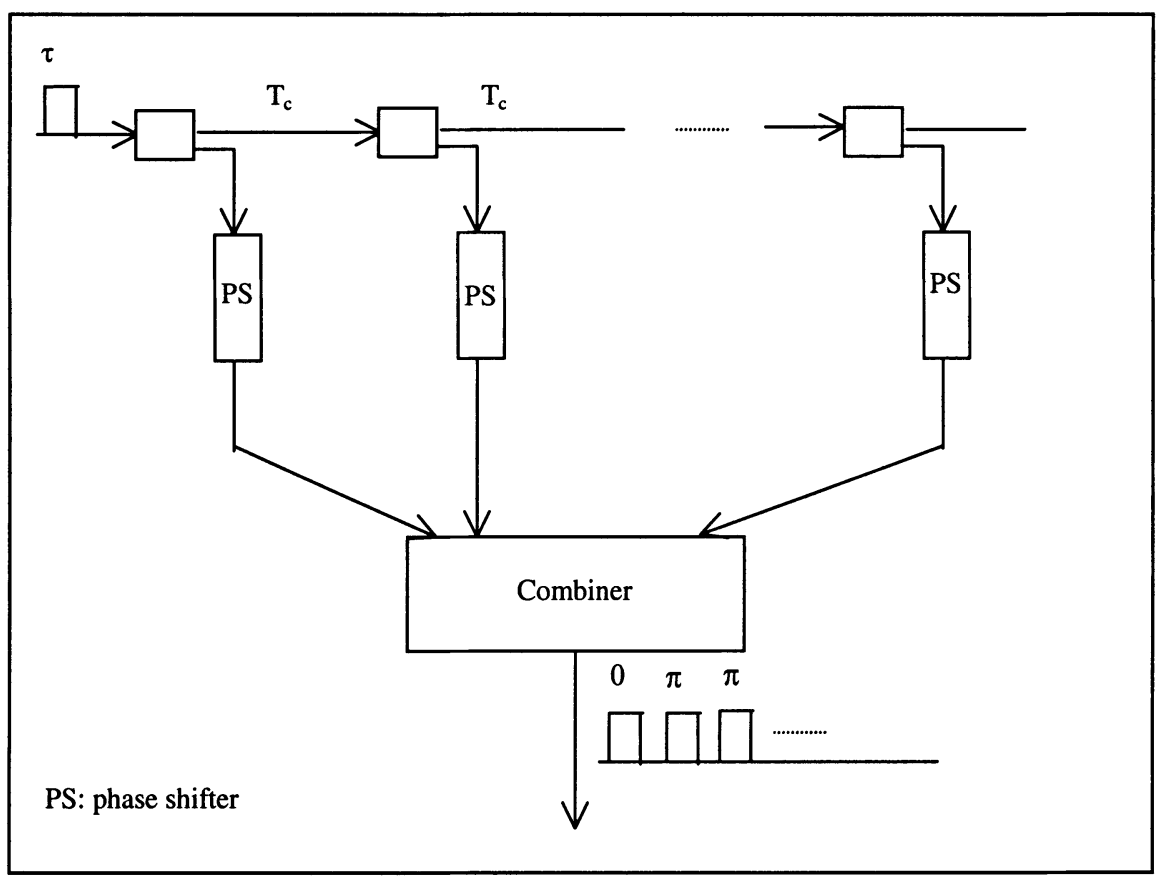

Figure 3. General structure of all optical encoder/decoder. 
The optical transmitted signal can then be written as

$$
y(t)=\sqrt{2 P_{T}}\left(\sum_{k=-\infty}^{\infty} a_{k} c(t-k T) e^{j 2 \pi f_{0} t}+\sum_{k=-\infty}^{\infty}\left(1-a_{k}\right) c(t-k T) e^{j 2 \pi f_{1} t}\right)
$$

where $\left\{a_{k}\right\}$ is the unipolar data sequence, $f_{0}=c / \lambda_{0}, f_{1}=c / \lambda_{1}$ and $P_{T}$ the peak optical power.

The code waveform $c(t)$ is given by:

$c(t)=\sum_{j=0}^{N-1} c_{j} p_{\tau}\left(t-j T_{c}\right)$

where $p_{\tau}(t)$ is a rectangular pulse of duration $\tau$, and $\left\{c_{j}\right\}$ the bipolar $( \pm 1)$ code sequence.

The structure of the receiver is shown in Fig. 4. The optical received signal is splitted in two arms. An optical filter tuned to the optical frequency $f_{0}$, extracts the first component in the right hand side of (1), while the filter tuned to $f_{1}$, recovers the second term. An optical decoder matched to the encoder used in the transmitter, i.e. with the taps reversed, follows each optical filter. This gives at the sampling points $k T$, an intensity peak in the upper arm if the data symbol is a logical one and a peak in the lower arm if the data symbol is a logical 0 . Each decoder is followed by a photodetector and the difference applied to a decision device according to the principles of conventional non-coherent FSK receivers.

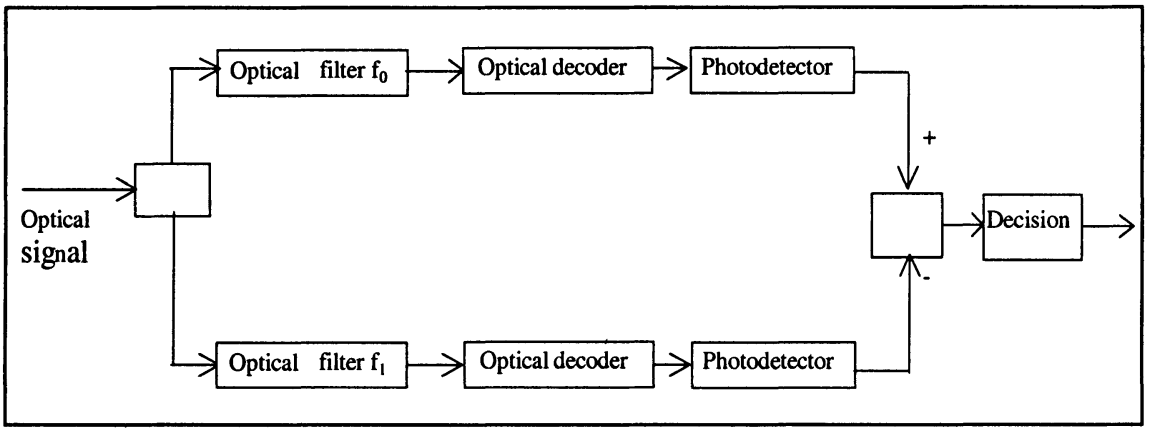

Figure 4. Block diagram of the receiver. 


\section{SYSTEM ANALYSIS}

In the analysis we shall consider the two cases of synchronous and asynchronous users In a synchronized network, there is some centralized control that provides the correct timing to each station so that the signals from the different stations arrive synchronous, i.e. time aligned at the central $\mathrm{NxN}$ optical coupler. In an unsynchronized network, there is no timing coordination between the stations and the signals arrive at the coupler with different timing alignments. A synchronized network may achieve a significantly better performance than an unsynchronized one since good codes are much easier to design with this restriction but it entails an extra complexity, and it may often preferable to set the network with uncoordinated users easing the management tasks.

We will consider without loss of generality the receiver for user 0 , and once we are interested in the performance limits imposed by the existence of multi-user interference, we will consider ideal components and neglect other sources of disturbance. The derivation assumes thus a high enough signal to noise ratio so that the system is multi-user interference limited.

Considering first the synchronized network, the optical received signal for $K$ simultaneous users is in complex notation

$$
r(t)=\sqrt{2 P_{R}} \sum_{i=0}^{K-1}\left(\sum_{k=-\infty}^{\infty} a_{k}^{(i)} c^{(i)}(t-k T) e^{j\left(2 \pi f_{0} t+\phi_{i}\right)} \sum_{k=-\infty}^{\infty}\left(1-a_{k}^{(i)}\right) c^{(i)}(t-k T) e^{j\left(2 \pi f_{1} t+\theta_{i}\right)}\right)
$$

where it is assumed that we have a perfect power control.

Let us consider that the undesired frequencies are completely eliminated by each of the optical filters and a code length of $N$. The complex envelope at the output of the matched decoders at time $t=T$ is given by

$$
\begin{aligned}
& r_{0}(T)=\alpha \sqrt{P_{R}}\left(a_{0}^{(0)} N E_{p}+\sum_{i=1}^{K-1} a_{0}^{(i)} e^{j \phi_{i}} \sum_{j=0}^{N-1} c_{j}^{(0)} c_{j}^{(i)} E_{p}\right) \\
& r_{1}(T)=\alpha \sqrt{P_{R}}\left(\left(1-a_{0}^{(0)}\right) N E_{p}+\sum_{i=1}^{K-1} a_{0}^{(i)} e^{j \theta_{i}} \sum_{j=0}^{N-1} c_{j}^{(0)} c_{j}^{(i)} E_{p}\right)
\end{aligned}
$$


$E_{p}=\int_{-\infty}^{\infty} p^{2}(t) d t, \quad$ is the energy of the elementary pulse, $\sum_{j=0}^{N-1} c_{j}^{(0)} c_{j}^{(i)}=R_{0, i}(0)$ the cross-correlation between the code of users 0 and $i$, with the sequences aligned, and $\alpha$ accounts for the losses of the splitter and filter.

The variable used for performing the decision about bit 0 is thus

$$
\begin{aligned}
\nu=\left|r_{0}(T)\right|^{2}-\left|r_{1}(T)\right|^{2}= & \alpha^{2} P_{R} E_{p}^{2}\left|a_{0}^{(0)} N+\sum_{i=1}^{K-1} a_{0}^{(i)} e^{j \phi_{i}} R_{0, i}(0)\right|^{2} \\
& -\alpha^{2} P_{R} E_{p}^{2}\left|\left(1-a_{0}^{(0)}\right) N+\sum_{i=1}^{K-1}\left(1-a_{0}^{(i)}\right) e^{j \phi_{i}} R_{0, i}(0)\right|^{2}
\end{aligned}
$$

From (5), we conclude that if the codes are orthogonal $\left(R_{0, i}(0)=, \forall i \neq 0\right)$, then the system is multi-user interference free, and the decision variable is simply proportional to $b_{0}^{(0)}=2 a_{0}^{(0)}-1$, requiring a threshold comparison against 0. Bipolar orthogonal codes can be constructed using Hadamard matrices through the iterative procedure

$$
\begin{aligned}
& \mathbf{H}(0)=[1] \\
& \mathbf{H}(n)=\left[\begin{array}{cc}
\mathbf{H}(n-1) & \mathbf{H}(n-1) \\
\mathbf{H}(n-1) & -\mathbf{H}(n-1)
\end{array}\right]
\end{aligned}
$$

leading to a total of $2^{n}$ orthogonal codes with length $N=2^{n}$, and consequently a synchronous network can support $2^{n}$ simultaneous users.

Let us now consider a network with uncoordinated users. The signals from the different stations arrive at the optical coupler with different delays and consequently the optical signal at receiver 0 , is

$r(t)=\sqrt{2 P_{R}} \sum_{i=0}^{K-1}\left(\sum_{k=-\infty}^{\infty} a_{k}^{(i)} c^{(i)}\left(t-k T-\tau_{i}\right) e^{j\left(2 \pi f_{0} t+\phi_{i}\right)} \sum_{k=-\infty}^{\infty}\left(1-a_{k}^{(i)}\right) c^{(i)}\left(t-k T-\tau_{i}\right) e^{j\left(2 \pi f_{1} t+\theta_{i}\right)}\right)(7)$

where $\tau_{i}$ is the delay of signal $i$.

The derivation the bit error rate (BER) performance is done in Appendix A. Assuming that the code sequences can be considered as true random binary sequences, one gets 
$p_{b}=\frac{1}{2} e^{-\frac{3 N}{2(K-1)}}$

Equation (8) is for well designed bipolar codes such as Kasami or Gold, a conservative upper bound, since the auto-correlation and cross-correlation properties of these codes are better than those of pure random sequences. Comparing against the performance obtained with a fully coherent PSK system, (8) represents a degradation slightly higher than $3 \mathrm{~dB}$, i.e. the number of simultaneous users is reduced by a factor of 2 due to the non-coherent nature of the detection process.

\section{NUMERICAL RESULTS AND COMPARISONS}

To illustrate expected performance of the system in an unsynchronized network, we plot in Fig. 5, the bit error rate as a function of the number of users $K$, for codes lengths $31,63,127$ and 255 , which are the natural length of Gold codes. The results show that for a target of $10^{-9}$ for the BER the maximum number of simultaneous users is slightly under $10 \%$.

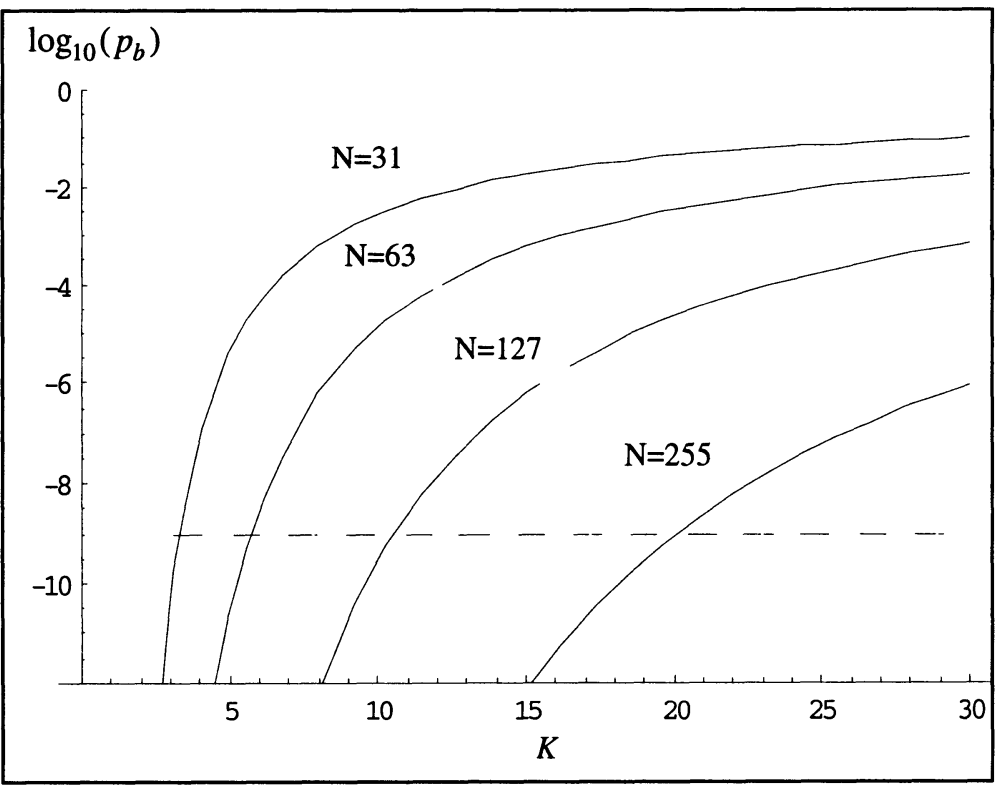

Figure 5. Bit Error rate as a function of the number of users in an asynchronous network. 
To compare the proposed system against the more conventional approaches based on unipolar codes, we consider the expected throughput for a given BER target. Let us define the throughput as the maximum allowable aggregate bit rate per channel usage, where the channel usage is the chip slot duration $T_{c}$.

In a synchronous network with orthogonal codes of length $N=2^{n}$ as described in section 3 , the optical channel can be shared by $2^{n}$ users each with a bit rate $1 / 2^{n} T_{c}$. The aggregate bit rate is thus $1 / T_{c}$ giving the throughput,

$$
\eta_{s, b}=1
$$

In the asynchronous network the system is not interference free, and we have to specify a BER target to define the system throughput. Let be $L$ the argument of the exponential in (8) that guarantees the required BER, then the maximum number of simultaneous users is

$$
K_{\max }=1+\frac{3 N}{2 L}
$$

and the throughput is

$$
\eta_{a s, b}=\frac{1}{N}+\frac{3}{2 L}
$$

To compare (9) and (11) against the conventional use of unipolar codes in optical communications, we consider the results of [3] for asynchronous networks and [5] for the synchronous case. In [3] the authors considered unipolar prime codes, and in [5] a modification of them consisting in an extended set including the original prime codes and the corresponding shifted versions. Prime codes, are binary unipolar sequences of weight $P$ and length $N=P^{2}$, with $P$ being a prime. The bit error rate in asynchronous networks, obtained with this family of codes, was after some approximations found to be [3] 


$$
p_{b}=Q\left(\frac{P}{\sqrt{1.16(K-1)}}\right)
$$

and for synchronous networks [5]

$$
p_{b}=Q\left(\frac{P}{\sqrt{K-1}}\right)
$$

where $Q(x)=\frac{1}{\sqrt{2 \pi}} \int_{x}^{\infty} e^{-u^{2} / 2} d u$

Let $\gamma$ bet the argument of $\mathrm{Q}($.$) that guarantees the BER target. From (12)$ and (13) he maximum number of simultaneous users is

$$
\left\{\begin{array}{cc}
K_{\max }=1+\frac{P^{2}}{1.16 \gamma^{2}} & \text { Asynchronous network } \\
K_{\max }=1+\frac{P^{2}}{\gamma^{2}} & \text { Synchronous network }
\end{array}\right.
$$

and the throughput

$$
\begin{array}{ll}
\eta_{a s, u}=\frac{1}{P^{2}}+\frac{1}{1.16 \gamma^{2}} & \text { Asynchronous network } \\
\eta_{s, u}=\frac{1}{P^{2}}+\frac{1}{\gamma^{2}} & \text { Synchronous network }
\end{array}
$$

Using (9), (11) and (14), we get in the limiting case of long codes, and for BER target $p_{b}=10^{-9}$, the following results: 
- Unipolar synchronous: $\eta \rightarrow 2.8 \%$

- Proposed scheme asynchronous: $\eta \rightarrow 7.5 \%$

- Proposed scheme asynchronous: $\eta=1$.

We note that with unipolar codes there are no significant throughput improvements when going from an asynchronous to a synchronous network. This is an expected result, since the motivation of the author in defining the modified prime codes was to increase the number of available code sequences while maintaining the cross-correlation limits. The use of the proposed scheme with bipolar codes provides a dramatic improvement if the network is synchronous. For the asynchronous case, the improvement is far less spectacular but still non-negligible, and in fact with well-designed codes such as Kasami or Gold, we expect a slightly better improvement than the one obtained assuming random sequences.

\section{CONCLUSIONS}

In this communication we have proposed and analyzed a transmission / reception scheme that allows the combination of non-coherent detection and bipolar codes in optical CDMA. The scheme relies on the optical bipolar coders / decoders proposed in [2], and is basically a direct-sequence spread spectrum transmission system with a non-coherent FSK modulation. The use of non-coherent detection eliminates the need of the complex and sensitive phase control loops, essential in any coherent scheme, and represents thus an important step towards the simplification of receivers. The analysis of the system was done both for synchronous and asynchronous users in the network. The results show that the main feature of bipolar codes arises in synchronous networks, where the use of orthogonal bipolar codes allows a dramatic improvement in the throughput when compared against the conventional sequences. With asynchronous users, there is still some significant improvement although much less pronounced that in the case of synchronous networks.

\section{APPENDIX A}

Let us consider the signal defined by (7). We shall without loss of generality consider the detection of user 0 , and assume the delay and phase shifts for this signal to be the reference, i.e. $\tau_{0}=0, \phi_{0}=0, \theta_{0}=0$. The signal at the output of the matched filter at $T$, is (omitting the factor $\alpha \sqrt{P_{R}}$ ) 


$$
\begin{aligned}
& r_{0}(T)=\alpha \sqrt{P_{R}}\left(a_{0}^{(0)} N E_{p}+\sum_{i=1}^{K-1}\left(a_{-1}^{(i)} R_{0 i}\left(\tau_{i}\right)+a_{0}^{(i)} \hat{R}_{0 i}\left(\tau_{i}\right)\right) e^{j \phi_{i}}\right) \\
& r_{1}(T)=\alpha \sqrt{P_{R}}\left(\left(1-a_{0}^{(0)}\right) N E_{p}+\sum_{i=1}^{K-1}\left(\left(1-a_{-1}^{(i)}\right) R_{0 i}\left(\tau_{i}\right)+\left(1-a_{0}^{(i)}\right) \hat{R}_{0 i}\left(\tau_{i}\right)\right) e^{j \theta_{i}}\right)
\end{aligned}
$$

where

$$
\begin{aligned}
& R_{0 i}\left(\tau_{i}\right)=E_{p 1}^{(i)} \sum_{j=0}^{r} c_{j}^{(i)} c_{N+j-r-1}^{(i)}+E_{p 2}^{(i)} \sum_{j=0}^{r-1} c_{j}^{(i)} c_{N+j-r}^{(i)} \\
& \hat{R}_{0 i}\left(\tau_{i}\right)=E_{p 1}^{(i)} \sum_{j=r+1}^{N-1} c_{j}^{(i)} c_{j-r-1}^{(i)}+E_{p 2}^{(i)} \sum_{j=r}^{N-1} c_{j}^{(i)} c_{j-r}^{(i)}
\end{aligned}
$$

with $\tau_{i}=r T_{c}+\varepsilon_{i}\left(0 \leq \varepsilon_{i}<T_{c}\right)$ and

$$
\begin{aligned}
& E_{p 1}^{(i)}=\int_{0}^{\varepsilon_{i}} p_{\tau}(t) p_{\tau}\left(t-\varepsilon_{i}\right) d t \\
& E_{p 1}^{(i)}=\int_{\varepsilon_{i}}^{T_{c}} p_{\tau}(t) p_{\tau}\left(t-\varepsilon_{i}\right) d t
\end{aligned}
$$

To obtain tractable results we assume that the multi-user interference affecting the components $a_{o}^{(0)} N E_{p}$ and $\left(1-a_{o}^{(0)}\right) N E_{p}$ in (A.2) can be modeled as a complex Gaussian process. This is a reasonable assumption for a moderate to high number of simultaneous users. Furthermore we shall use the following assumptions:

- The phase shifts $\phi_{i}$ and $\theta_{i}$ are uniformly distributed in the interval $\lfloor 0,2 \pi\rfloor$ and are uncorrelated.

- The time delays $\tau_{i}$, are uniformly distributed in the interval $\lfloor 0, T\rfloor$ and independent.

- The code sequences $\left\{c_{j}^{(i)}\right\}$ are assumed to be independent sequences of i.i.d. random variables taking the values 1 and -1 with equal probability. 
The duration of $p_{t}(t)$ is $T_{c}$.

Let us define the real and imaginary parts of the interferences as

$$
\begin{aligned}
& I_{0}(T)=\sum_{i=1}^{K-1}\left(a_{-1}^{(i)} R_{0 i}\left(\tau_{i}\right)+a_{0}^{(i)} \hat{R}_{0 i}\left(\tau_{i}\right)\right) e^{j \phi_{i}}=n_{c 1}+j n_{s 1} \\
& I_{1}(T)=\sum_{i=1}^{K-1}\left(\left(1-a_{-1}^{(i)}\right) R_{0 i}\left(\tau_{i}\right)+\left(1-a_{0}^{(i)}\right) \hat{R}_{0 i}\left(\tau_{i}\right)\right) e^{j \phi_{i}}=n_{c 2}+j n_{s 2}
\end{aligned}
$$

It is easy to verify that with the assumptions pointed out we have

$$
\left(n_{c i}, n_{s i}\right) \propto N\left(0,(K-1) T_{c}^{2}\right) \quad i=0,1
$$

Because of the symmetry of the problem, we may assume without loss of generality $a_{0}^{(0)}=1$, and compute the probability of bit error for this case. We get an error decision if

$$
\left|N T_{c}+n_{c 0}+j n_{s 0}\right|^{2}<\left|n_{c 1}+j n_{s 1}\right|^{2}
$$

and then using the well-known results on the probability of a Rice random variable exceeding another one [14] we obtain

$$
p_{b}=\frac{1}{2} e^{-\frac{3 N}{2(K-1)}}
$$

\section{REFERENCES}

[1] Special Issue on Multiwavelength Optical Technology and Networks, IEEE J. Light.., vol. 14, June 1996.

[2]K. Kitayama, "Code division multiplexing lightwave networks based upon optical code conversion”, ”, IEEE J. Select. Areas Commun., vol. 14, pp. 1309-1319, Sept. 1998.

[3] P. R. Prucnal, M. A. Santoro and T. R. Fan, "Spread-spectrum fiber optic local area network using optical processing", IEEE Journal of Light. Tech., pp.547-554, May 1986.

[4] J. A. Salehi and C. A. Brackett, "Code division multiple access techniques in optical fiber networks - Part II: System performance analysis", IEEE Trans. Commun, pp.834-842, Aug. 1989. 
[5] W. C. Kwong, P. A. Perrier and P. R. Prucnal, "Performance comparison of asynchronous code-division multiple-access techniques for fiber-optic local area networks", IEEE Trans. Commun, pp.1625-1634, Nov. 1991.

[7] J. K. Tang and K. Ben Letaief, "Optical CDMA communication systems with multi-user and blind detection", IEEE Trans. Commun., vol. 47, pp. 1211-1217, Aug. 1999.

[8] M. E. Marhic, "Coherent optical networks", Journal of Light. Tech., pp. 854-864, May/June 1993.

[9] N. Wada and K. Kitayama "A 10Gb/s optical code division multiplexing using 8-chip optical bipolar code and coherent detection", Journal of Light. Tech., pp. 1758-1769, Oct. 1999.

[10] W. Huang and I. Andonovic, "Coherent optical pulse CDMA systems based on coherent correlation detection”, IEEE Trans. Com, pp.261-271, Feb. 1999.

[11] F. Chung, J. Salehi and V. Wei, "Optical orthogonal codes: design, analysis and applications”, IEEE Trans, Inf. Theory, pp. 595-604, May 1989.

[12] A. S. Holmes and R. Syms, "All-optical codes using "quasi-prime" codes", IEEE Journal of Light. Tech., pp.279-286, Feb 1992.

[13] M. Chang and M. E. Maric, "Fiber-optic ladder networks for inverse decoding coherent CDMA”, Journal of Light. Tech., pp. 1952-1962, 1992.

[14] J. G. Proakis, Digital Communications, MacGraw Hill 1989. 